\title{
HYBRIDER HANDEL ALS ANTWORT AUF SMART SHOPPING
}

\author{
Lukas Zühlke, Michael Stolle, Bertil Haack
}

\section{Zusammenfassung}

Smart Shopping bedeutet, dass moderne Kunden eine Vielzahl verschiedener „Kanäle“ für den Kaufabschluss bei einer gewünschten Ware nutzen. Dieses Phänomen erstreckt sich über den gesamten Kaufprozess, vom ersten Auftreten eines Bedürfnisses bis zur Nachbetreuung des Kunden durch den Händler. In Zeiten des wachsenden E-Commerce und sich neu entwickelndem M-Commerce bildet sich jedoch bereits eine neue Form des zukünftigen Handels heraus, der hybride Handel. Die vorliegende Arbeit erörtert, warum hybrider Handel die Antwort auf Smart Shopping ist. Dabei wird zunächst das Smart Shopping erläutert (Kapitel 2) und daraus wird hybrider Handel abgeleitet und definiert (Kapitel 3). Im weiteren Verlauf der Arbeit werden Beispiele, Chancen und Risiken des hybriden Handels aufgezeigt und diskutiert (Kapitel 4). Fazit und Ausblick runden die Ausführungen ab (Kapitel 5).

\section{Abstract}

Smart Shopping means that today's customer use a variety of channels for the transaction of a desired product. This phenomenon bears over the whole process of purchasing, from the upcoming desire, until the aftercare operations. In times of growing e-commerce and arising $\mathrm{m}$-commerce, a new form of commerce is evolving, it's called hybrid commerce. In the present article the authors will discuss the topic, why hybrid commerce is the response to smart shopping. Therefore the term smart shopping will be elucidated (chapter 2) and from this term the definition of hybrid commerce will be extrapolated (chapter 3 ). Furthermore, examples, chances and risks of hybrid commerce will be revealed and discussed (chapter 4). The paper ends with a summary and forward looking statement (chapter 5).

\section{EINLEITUNG}

Aktuelle Untersuchungen zeigen, dass das Einkaufen einen weitreichenden Wandel erfährt. Während früher zwangsläufig der offline-Einkauf im Ladengeschäft dominierte und Schritt für Schritt durch den Versandhandel oder auch das Teleshopping ergänzt bzw. teilweise abgelöst wurde, werden Käufe heute in erheblichem Maße online am Computer bzw. mobil per Smartphone oder Tablet-PC getätigt. (Smart Shopping o. J.) Dabei wird erwartet, dass in "etwa 5 Jahren [...] rund $80 \%$ der Waren im deutschen eCommerce-Handel über mobiles Shopping verkauft" werden. (Smart Shopping o. J.: 8)

Entsprechend scheinen Beispiele wie die nicht mehr existierenden Versandhändler Neckermann und Quelle und der in schweres Fahrwasser geratene Karstadt-Konzern die Vermutung zu stützen, dass zukünftig nur noch die Handelsunternehmen erfolgreich am Markt agieren können, die sich mindestens auf den Einkaufskanal „online“, insbesondere aber auf den Kanal „mobil“ konzentrieren.
Diese Annahme entspricht so jedoch nicht der Realität. Tatsächlich deutet sich seitens der Verbraucher eine Entwicklung in Richtung Smart Shopping an (Abbildung 1): Abhängig vom gewünschten Gut nutzen Smart Shopper jede mögliche Kombination der Kanäle offline, online und mobil, um sich über das interessierende Produkt zu informieren, es auszuwählen und schließlich zu bezahlen. (Smart Shopping o. J.)
Folglich darf eher vermutet werden, dass die Berücksichtigung des Smart Shoppings ein kritischer Erfolgsfaktor für den Handel ist.

Was genau bedeutet dies für den Handel? Wie sollte er auf Smart Shopping reagieren? Welche organisatorischen, technischen und sonstigen Maßnahmen sind erforderlich?

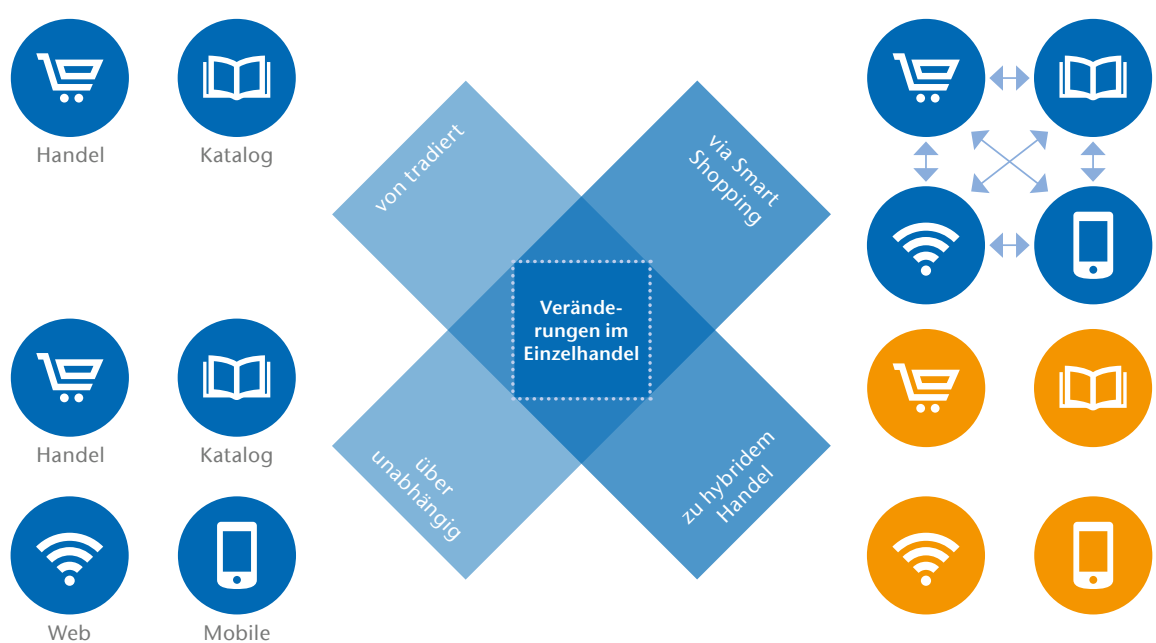

Abb. 1) Das veränderte Geschäftsmodell für den Einzelhandel (eigene Darstellung in Anlehnung an Profitable growth in a digital age 2013). 
Die vorliegende Arbeit gibt Antworten auf diese Fragen - teilweise ausgerichtet auf den Textileinzelhandel in Deutschland. Zunächst wird dazu das klassische Einkaufsverhalten der Kaufinteressenten sowie der Kunden beschrieben und es werden Aussagen zum Smart Shopping als modernem Einkaufsverhalten getroffen (Kapitel 2). Als Reaktion hierauf wird das Konzept des hybriden Handels entworfen und exemplarisch erörtert (Kapitel 3). Weiterhin werden bereits existierende Beispiele diskutiert, die zeigen, inwieweit Ansätze hybriden Handels aktuell Eingang in den Textileinzelhandel gefunden haben (Kapitel 4). Fazit und Ausblick beschließen die Ausführungen (Kapitel 5).

\section{SMART SHOPPING}

Einkaufsprozesse vollziehen sich in drei Phasen (Abbildung 2):

- Vorkaufsphase

- Kaufphase

Nachkaufphase ausführen und dafür Sportschuhe erwerben möchte. In diesem Fall beginnt der Kunde zumeist mit der Suche nach Informationen über das gewünschte Produkt auf Basis eigener Erfahrungen, der Erfahrungen ihm bekannter Personen oder auch mit Hilfe von Werbematerialien. Hat er genügend Informationen gesammelt, wertet er die Ergebnisse seiner Nachforschungen aus und evaluiert die Kaufmöglichkeiten. (Foscht et al. 2007: $161 \mathrm{ff}$.)

Kommt der potenzielle Käufer dabei zu für ihn positiven Ergebnissen seiner Suche, können wir annehmen, dass er die Intention entwickelt, einen bestimmten Sportschuh zu kaufen. Damit beginnt der Übergang zur Kaufphase, in der sich der tatsächliche Kauf vollzieht und der potenzielle Käufer zum (tatsächlichen) Käufer wird.

Beim offline-Einkauf kommt der Zeitspanne zwischen dem Zustand „Kaufintention entwickelt“ und „Kaufintention kann erfüllt werden" eine erhebliche Bedeutung zu. Je länger sie dauert, beispielsweise weil sich der potenzielle Käufer am Wochenende informiert hat, aber erst nach dem startet der gesamte Prozess erneut und der Kauf wird unter Umständen sogar verworfen. (Foscht et al. 2007: 87)

Ist es zum Kauf gekommen, die Ware also gegen Geld übergeben, beginnt die Nachkaufphase des erworbenen Produktes. Diese ist einerseits durch die (erwartete) Nutzung, etwa der fraglichen Sportschuhe, durch den Käufer gekennzeichnet. Andererseits ist es die Phase, in der wesentliche Maßnahmen zum Aufbau einer langfristigen Kundenbindung Platz greifen können. Diese Betreuung des Kunden kann in einem Angebot an Rückgaberechten, Serviceleistungen bei Reklamationen sowie in Anregungen zu Nachkäufen bestehen. Im Textileinzelhandel beschränkt sie sich meist auf die Abwicklung von Reklamationen und Beschwerden. (Broeckelmann 2010: $38 \mathrm{ff}$.)

Dieses klassische Einkaufsverhalten hat sich zusammen mit dem Internet und dessen Nutzung als Verkaufsfläche rasant weiterentwickelt. Modernes Einkaufsverhalten ist in allen Einkaufsphasen vom Internet und seinen Möglichkeiten beeinflusst:

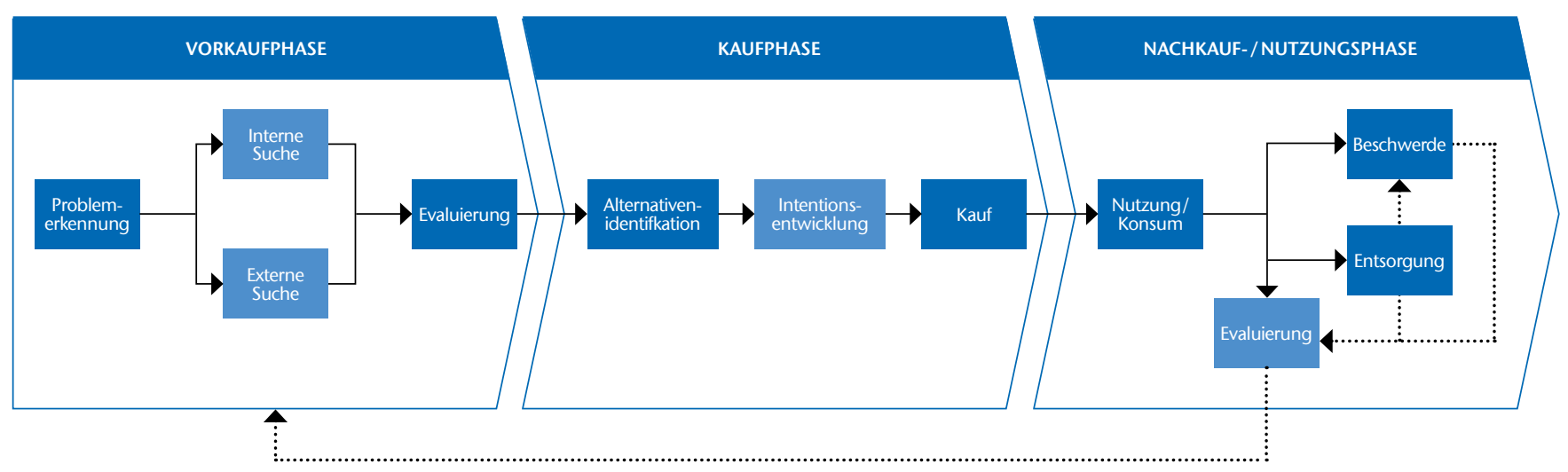

Abb. 2) Der Kaufprozess (Der Kaufprozess o. J.).

Klassisch, d.h. beim offline-Einkauf in einem Ladengeschäft, laufen diese Phasen in der Regel wie folgt ab:

Die Vorkaufphase setzt ein, wenn bei einem potenziellen Käufer ein Bedürfnis nach einem bestimmten Produkt entsteht. Das kann beispielsweise der Fall sein, wenn er eine neue Sportart
Wochenende einkaufen kann, desto eher kann es sein, dass der potenzielle Käufer seine Kaufintention verliert und vom Erwerb etwa der Sportschuhe absieht. Ursache können unter anderem zusätzliche Informationen sein, die der Interessent in diesem Zeitabschnitt erhält und die ihn zu einer erneuten Evaluation veranlassen. In diesem Fall
In Zeiten, in denen jeder Mensch Informationen im Internet nicht nur sondieren, sondern auch selbst weltweit zur Verfügung stellen kann, ist die Suche nach Fakten zu einem Produkt um ein Vielfaches leichter als früher ohne Internet. Zu den klassischen Informationsquellen gesellt sich ein Erfahrungsschatz der Kunden, die sich in 
Foren und Weblogs zu den einzelnen Produkten austauschen. Die Vorkaufphase erfährt dadurch bereits deutliche Veränderungen.

Die wesentliche Neuerung im Informationsprozess ist jedoch, dass jeder Besitzer eines mobilen Endgerätes die Chance hat, sich mobil zu informieren und dann zu entscheiden, wie und wo der eigentliche Kauf stattfindet.

Während sich das klassische Einkaufsverhalten im Einzelhandel dadurch manifestiert, dass sich ein Kunde offline, also im Laden, informiert und auch offline, im besten Fall im selben Laden, einkauft, haben Interessenten nun zum Beispiel die Möglichkeit, sich noch vor Ort im Ladengeschäft via Internet über möglicherweise günstigere oder qualitativ hochwertigere Konkurrenzprodukte zu informieren und diese bei einem anderen Anbieter zu erwerben. Hiervon macht jeder dritte potenzielle Kunde Gebrauch. (Mobile Informationssuche wird für den Einkauf wichtiger 2012) Potenzielle Kunden nutzen aber auch alternative Vorgehensweisen: So bietet sich speziell beim Textileinzelhandel an, sich offline $z u$ informieren und online zu kaufen. Dies bedeutet, dass der potenzielle Kunde die Ware nur noch im Laden testet, um Stoff, Größe und Haptik auszuprobieren. Der tatsächlicher Kauf und somit die Umsatzgenerierung für den Händler erfolgen dann jedoch bequem von zu Hause im Internet oder mobil per Smartphone oder Tablet-PC. Andere Kaufinteressenten informieren sich mobil auf ihrem Smartphone und kaufen im Laden, wenn sie dort den besten Preis bekommen oder sie die Ware noch am selben Tag benötigen. Wieder andere potenzielle Kunden informieren sich online über Produkte und kaufen diese dann auch sofort online ein. (Smart Shopping o. J.).

Modernes Einkaufsverhalten - Smart Shopping - ist damit dadurch charakterisiert, dass die Käuferinnen und Käufer - Smart Shopper - abhängig von der gewünschten Ware - z. B. Textilien oder Lebensmittel - jede mögliche Kombination der Einkaufskanäle offline, online und mobil nutzen, um sich über das interessierende Produkt

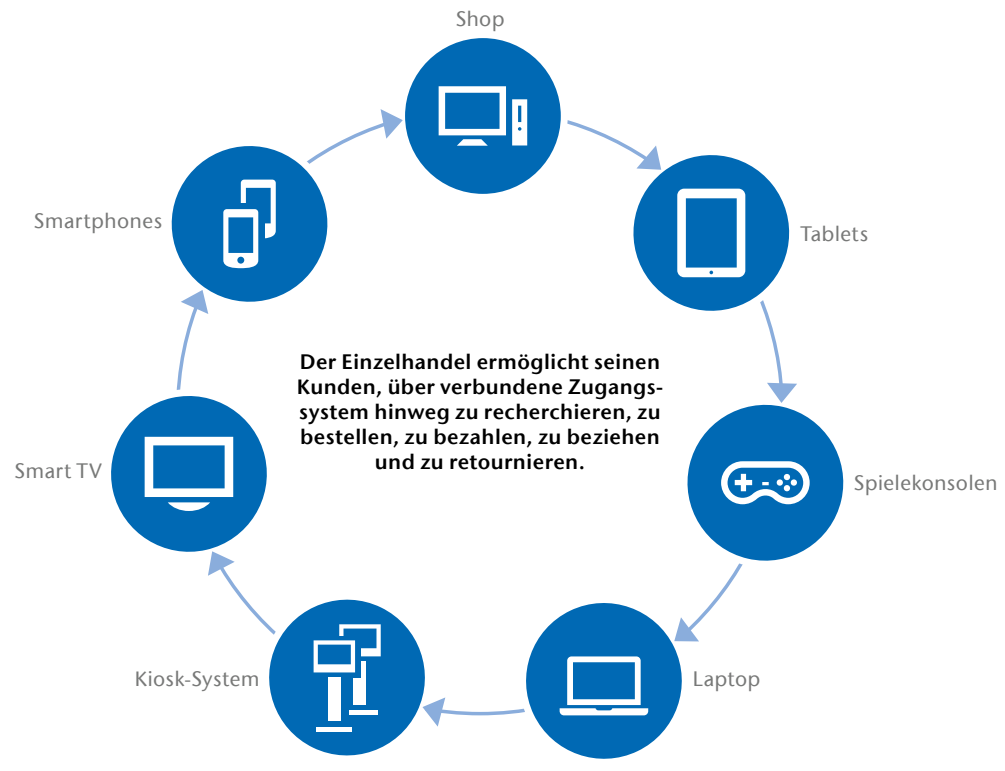

Abb. 3) Smart Shopping durch hybriden Handel (eigene Darstellung in Anlehnung an Marsden 2013).

zu informieren, es auszuwählen und schließlich zu bezahlen. (Smart Shopping o. J.).

Die Entscheidung des Smart Shoppers für sein jeweiliges Vorgehen basiert wesentlich auf den Vorteilen, die er dabei hat. Während das mobile Kaufen die Möglichkeit eröffnet, immer und überall Modeartikel zu erwerben, hat das stationäre Kaufen den Vorteil, dass der Interessent den Artikel anfassen und fühlen kann, was für viele immer noch ein wichtiger Bestandteil des Einkaufprozesses ist. In anderen Fällen entscheiden Zeitvorteile oder auch die bequeme Zahlung mit Kreditkarte oder Smartphone.

Wie kann der Handel auf das Einkaufsverhalten der Smart Shopper reagieren? Unsere Antwort lautet: durch hybriden Handel.

\section{HYBRIDER HANDEL}

Unter Multichannel-Handel wird verstanden, dass ein und derselbe Händler mehrere voneinander getrennte Vertriebskanäle für seine Waren zur Verfügung stellt, die von den Kaufinteressenten und Käufern als Einkaufskanäle genutzt werden können.

Entscheidendes Kriterium dafür, ob ein Händler Multichannel-Handel betreibt, ist, dass jeder einzelne Kanal auch ohne den anderen lebensfähig sein muss und Umsatz für den Händ- ler generieren kann, dass also mehrere voneinander unabhängige Vertriebskanäle existieren.

Anders ausgedrückt: MultichannelHandel ist durch zwei Bedingungen gekennzeichnet. Beim MultichannelHandel kann ein potenzieller Käufer einerseits einen Vertriebskanal aus einer Menge verschiedener Kanäle für seinen Kauf auswählen. Andererseits muss der Kauf dann vollständig im gewählten Kanal abgewickelt werden, ohne dass ein Zugriff auf Informationen oder Ressourcen eines anderen Kanals erforderlich ist. (Zühlke 2013: 5).

Multichannel-Handel ist damit keine Antwort auf das Einkaufsverhalten der Smart Shopper, die im Extremfall zu beliebigen Zeitpunkten im Einkaufsprozess in beliebige andere verfügbare Vertriebs- bzw. Einkaufskanäle wechseln.

Entsprechend muss der Ansatz „Multichannel-Handel“ modifiziert werden, so dass er jegliche sinnvolle Verknüpfung der Vertriebskanäle zulässt. Wir kommen damit zum Konzept des „hybriden Handels“:

Hybrider Handel ist die Verknüpfung von mindestens zwei verschiedenen Absatzkanälen im Einzelhandel. Dabei ist entscheidend, dass sowohl der Händler als auch der Kunde die Möglichkeit haben, während des Verkaufsund Kaufprozesses sinnvoll zwischen den Kanälen zu wechseln beziehungs- 
weise Informationen aus den unterschiedlichen Kanälen miteinander zu verknüpfen.

Diese Art des hybriden Handels ist eine Erweiterung des MultichannelHandels: Einerseits ist MultichannelHandel Teil des hybriden Handels, weil jeder hybride Verkaufsprozess über einen ausgewählten Vertriebskanal abgewickelt werden kann und nicht über mehrere Kanäle abgewickelt werden muss. Andererseits geht hybrider Handel über den Multichannel-Handel hinaus, da sowohl der Kaufinteressent als auch der Händler mehrere Kanäle in einem Verkaufsprozess kombinieren kann und jeder Kanal Informationen für weitere Kanäle bereitstellt. Der Händler kann somit zum Beispiel Informationen, die er via Online-Handel über seine Kunden gewinnt, auch offline im stationären Ladengeschäft nutzen. Umgekehrt ist Entsprechendes auch den Kaufinteressenten und Kunden möglich. (Zühlke 2013: 5).

Entscheidend für den Erfolg des hybriden Handels ist, dass der Händler die Vorteile erkennt und für sich nutzt. Es ist wichtig, dass er ein ganzheitliches hybrides Konzept realisiert, das alle drei Phasen des Kaufprozesses abdeckt. Die Umsetzung eines derart weitgehenden Konzeptes bedeutet, dass händlerseitig umfangreiche technische und organisatorische Maßnahmen geplant und durchgeführt werden müssen. Dabei müssen auch die Mitarbeiterinnen und Mitarbeiter des jeweiligen Unternehmens „mitgenommen werden“. (Zühlke 2013: 33)

Exemplarisch seien folgende sinnvollen Bausteine eines hybriden Konzeptes genannt (Zühlke 2013: 5ff.):

- Hybrider Handel baut auf mehreren Kanälen auf, die dem Interessenten oder Kunden zur Wahl gestellt werden. Allein der Interessent oder Kunde entscheidet, welchen der verfügbaren Kanäle er nutzt, um sich über ein Produkt zu informieren bzw. es zu erwerben. Hat der Interessent beispielsweise die Vorkaufphase abgeschlossen und sämtliche Informationen gesammelt, muss es ihm in der Kaufphase möglich sein, einen anderen Kanal zu wählen.
- Hybrider Handel erfordert sowohl eine passende Homepage des Händlers als auch eine Applikation für den mobilen Gebrauch. Im besten Fall sind beide ähnlich aufgebaut und haben dasselbe Design sowie dieselbe Navigation. Dies kann eine mit erheblichen Kosten für den Händler verbundene Forderung sein. Möglicherweise bieten aber Angebote wie das von Couch Commerce die Chance, zügig und preiswürdig zu einer passenden Lösung zu kommen. (Wandeln Sie Ihren Onlineshop 2013)

- Im Falle von Textilien haben potenzielle Kunden oftmals den Wunsch, sich die Ware vor dem Erwerb anzuschauen und sie anzuprobieren. Es kann auch sein, dass das Kleidungsstück noch am selben Tag benötigt wird. Hybrider Handel lässt hier neben online- und mobil-Kanal auch eine offline-Einkaufsmöglichkeit - ein Geschäft oder ein Kaufhaus - als Vertriebskanal sinnvoll erscheinen (vgl. Kapitel 4).

- Verkaufspersonal und -fläche im offline-Handel sollten ebenfalls auf Smart Shopper eingestellt sein. Neben den technischen sollten auch die personellen Voraussetzungen gegeben sein, um flexibel auf Smart Shopper und deren Einkaufsverhalten reagieren sowie beispielsweise die aus den über die Käufer verfügbaren Informationen resultierenden Synergien heben zu können (vgl. Kapitel 4).

- In der Nachkaufphase können so genannte Location-based-Services, „standortbezogene Dienste, die auf aktuelle Aufenthaltsposition abgestimmte Informationen zur Verfügung stellen“ (LocationBased-Services 2013), im Sinne der Kundenbindung genutzt werden (vgl. Kapitel 4).

Zusammengefasst bietet hybrider Handel dem Händler und dem Kunden die Möglichkeit, auf verschiendenen Vertriebs- bzw. Einkaufskanälen aktiv zu werden, die einerseits unabhängig voneinander, andererseits aber auch bestmöglich miteinander verknüpft sind. Für den Händler bedeutet dies zunächst eine Umstrukturierung bestehender Konzepte und Denkweisen sowie wahrscheinlich erhebliche Investitionen in seine Technik. Er kann jedoch eine höhere Kundenbindung und Umsätze über alle Kanäle und damit ökonomischen Nutzen erwarten. Für Kaufinteressenten und Kunden bestehen die Vorteile des hybriden Handels darin, dass sie volle Flexibilität besitzen und jeden Kanal auf dem Weg zum Kauf betreten und wieder verlassen können, womit hybrider Handel dem Einkaufsverhalten „Smart Shopping" voll gerecht wird.

\section{BEISPIELE FÜR HYBRIDEN HANDEL IM TEXTILEINZELHANDEL}

Obwohl das Phänomen des hybriden Handels erst beginnt, setzen bereits viele Textileinzelhändler in Deutschland auf Ideen, die nach der gegebenen Definition einen hybriden Handel darstellen.

Beispielsweise nimmt das Düsseldorfer Modelabel Jades24 sehr gezielt die Vernetzung der Kanäle in seine Verkaufsstrategie auf und nutzt somit bereits jetzt ein sehr umfassendes hybrides Konzept, welches Vorteile sowohl für den Kunden als auch für den Händler bietet. Die Idee von Jades 24 ist, dem Kunden jederzeit zu ermöglichen, die Ware, die in den beiden Filialen in Düsseldorf ausgestellt ist, zu sehen und zu kaufen.

Realisiert wird dieses Konzept zunächst mittels QR-Code-basierten Schaufenstern, die der Düsseldorfer Kundschaft auch nach Ladenschluss die Möglichkeit eröffnen, die im Schaufenster ausgestellte Mode per Applikation auf dem Smartphone zu scannen und zu erwerben.

Befindet sich ein Kunde jedoch nicht in Düsseldorf, so gibt es dennoch eine Chance für ihn, die Ware so zu sehen, wie sie im Schaufenster ausgestellt wird. Dieses von Jades24 entwickelte Windowshopping ist eine Verknüpfung von Komponenten des stationären Handels mit Bausteinen des OnlineVertriebs, indem online Fotografien der Aussstellungen aus den Schaufenstern abgebildet werden (Abbildung 4). 
Darüber hinaus finden hybride Ansätze auch auf der Verkaufsfläche der beiden Filialen erhebliche Beachtung. So nutzt Jades 24 gezielt Kundendaten, um die Kunden auf besondere Aktionen in den Stores wie zum Beispiel Modenschauen aufmerksam zu machen. Hier werden Location-based-Services eingesetzt, welche ein personenbezogenes und gezieltes Marketing für Jades24 ermöglichen. Sollte es der Fall sein, dass ein Kunde eine Ware, die er im Internet gesehen hat, nicht mehr im stationären Handel vorfindet, kann er die Ware jederzeit mittels iPads, die sich im Laden befinden, bequem in den Store oder nach Hause bestellen. (Jades24 2013).

Ein weiterer Vorreiter in hybriden Handelskonzepten im Textileinzelhandel in Deutschland ist die Firma Esprit. Mit der Implementierung einer Verfügbarkeitsprüfung von online entdeckter Mode gelingt ihr eine Besonderheit in verfügbar ist. Über eine Farbskala wird dem Kunden zusätzlich der Grad der Verfügbarkeit angezeigt. Im Falle einer Nichtverfügbarkeit werden die Adresse und die Telefonnummer des wiederum nächstgelegenen Stores angezeigt, um die Ware dort per Telefon bestellen zu können.

Hinzu kommt, dass die Verfügbarkeit differenziert für verschiedene Farbkombinationen und Größen des jeweiligen Artikels dargestellt wird. Dabei kann die genaue Passform in Erfahrung gebracht werden, und über die Auswertung von Erfahrungsberichten wird automatisch ein Feedback anderer Kunden gegeben, wie die Ware beim Tragen ausfällt.

Zusammengefasst kann der Onlinekunde demnach einen scheinbar stationären Besuch im Laden durch den Onlinehandel ersetzen, da sämtliche Informationen vorab verfügbar sind.

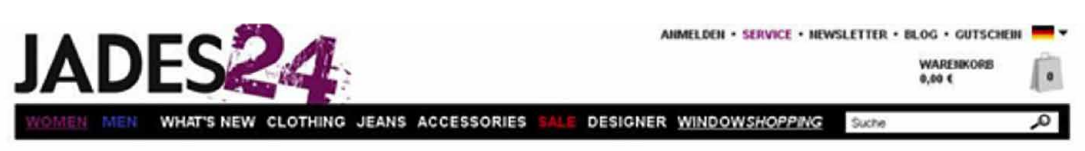

WINDOWSHOPPING
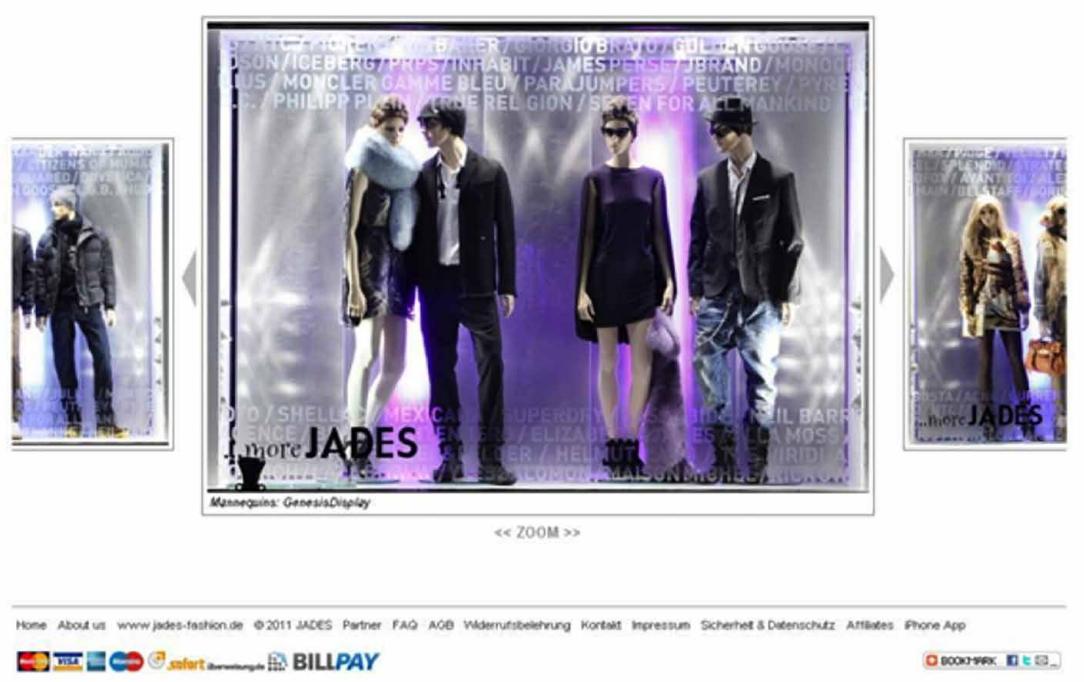

oposerex $n \in E$

Abb. 4) Jades24 Windowshopping (Stuttgarter Internetagentur LIGADIGITAL 2011).

der Verknüpfung von stationärem Offlinehandel und Onlinegeschäft.

Jeder Besucher der Website esprit.de hat die Möglichkeit, sich einen bestimmten Artikel online vorab auszusuchen und dann über eine Anzeige zu recherchieren, in welchem nächstgelegenen Laden das jeweilige Teil
Jedoch kann die finale Kaufentscheidung auch offline stattfinden, ohne langen Suchvorgang, in welchem Shop der Artikel verfügbar ist. (Esprit Global Image $\mathrm{GmbH}$ 2013).

Esprit hat aktuell etwa 7000000 Clubmitglieder. Das Unternehmen verfolgt die Überlegung, dass diejenigen Club- mitglieder den größten Umsatz generieren, die sowohl online als auch offline einkaufen. Daher versucht Esprit die Schnittstelle zwischen Online- und Offlineeinkauf so problemlos wie möglich für die Clubmitglieder zu gestalten. Dies zeigt sich speziell bei dem eigens für Esprit entwickelten Bonuspunktemodell. Jedes Clubglied bekommt für seinen Einkauf Bonuspunkte, welche es dann gegen Gutscheine oder Rabatte einlösen kann. Diese Form der Punktevergabe gilt als beliebtes Mittel im Marketing, um bei den Kunden einen stärkeren Kaufwillen zu erzeugen. Das Besondere am System von Esprit ist aber, dass ein Kunde, welcher normalerweise stets online einkauft, auch offline über den Gutschein verfügen und diesen dann im stationären Handel einlösen kann. Ebenfalls ist es für den klassischen Ladenkunden möglich, seine Bonuspunkte im Onlineshop von Esprit zu nutzen. Diese Form des Umgangs mit den Clubmitgliedern eröffnet dem Kunden die größtmögliche Flexibilität und bildet eine starke Verknüpfung zwischen den Basiselementen des stationären Handels und des Onlineversandhandels. (Esprit Global Image $\mathrm{GmbH}$ 2013).

Beim Einkauf im Onlinehandel steht der Kunde meist vor dem Problem, dass er sich für eine Kombination der Waren entschieden hat, aber nicht genau sagen kann, ob diese farblich und vom Schnitt zueinander passen könnten. Auch hier hat Esprit seit diesem Jahr eine Neuerung in den Onlinehandel implementiert. So ist es möglich, dass Kunden, die sich für verschiedene Artikel im Onlineshop interessieren, diese einer virtuellen Versuchsfigur per Drag \& Drop-System in einer virtuellen Umkleidekabine zur Anprobe geben können (Abbildung 5). Die Puppe kann dabei verschiedene Frisuren haben und somit dem Aussehen des Kunden angepasst werden. Ebenfalls ist es möglich, verschiedene Artikel übereinander zu tragen, so dass nicht nur ein Pullover und eine Hose getestet werden können, sondern auch ganze Outfits. (Esprit Global Image GmbH 2013).

\section{FAZIT UND AUSBLICK}

Das Einkaufsverhalten ist einem erheblichen Wandel unterworfen. 


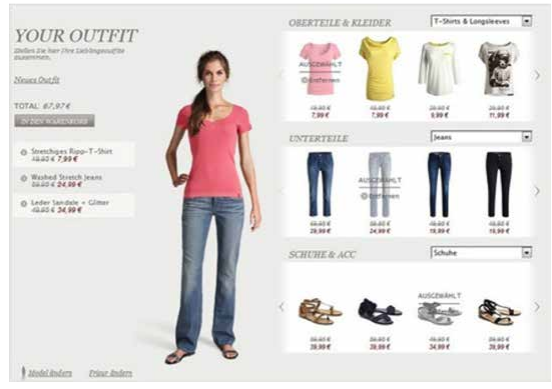

Abb. 5) Esprits virtuelle Umkleidekabine (Esprit Stylingstudio 2013).

Mehr noch als heute werden potenzielle Kunden zukünftig eine enge Verzahnung von offline-, online- und mobilem Einkauf nutzen. Das Konzept des hybriden Handels greift diese Anforderungen auf. Die Beispiele im Kapitel 4 zeigen, dass im deutschen Textileinzelhandel schon erste Ansätze für hybriden Handel gegeben sind. Der Handel beginnt bereits, sich darauf einzustellen, dass dem Kunden der Wechsel der Kanäle möglichst leicht gemacht werden muss, um somit Loyalität des Käufers zum Anbieter zu schaffen und dafür zu sorgen, dass der Kunde auch bei der jeweiligen Marke bleibt.

Damit stehen die Anbieter aber erst am Anfang einer Entwicklung. Entscheidend wird sein, das Onlinegeschäft nicht als Konkurrenz, sondern als Chance zu sehen. Die Textilunternehmen müssen einen ganzheitlichen Ansatz realisieren, der es einerseits ihnen ermöglicht, Informationen, die über den Kunden im einen Kanal benötigt werden, aus einem anderen Kanal zu bekommen, und der andererseits dem Kunden die Chance bietet, jederzeit in allen Phasen des Kaufprozesses den Kanal zu wechseln und dabei bei demselben Unternehmen zu bleiben.

Wenn es den Händlern gelingt, diese Kanalgrenzen auch unternehmensintern so niedrig wie möglich zu halten und dafür zu sorgen, dass die stationären Händler auch mit dem Onlinehandel zusammenarbeiten, kann der hybride Handel für alle Beteiligten gewinnbringend sein. Wenn dies nicht geschieht, besteht die Gefahr darin, dass der Kunde die stationären Läden mehr als Showrooms sieht, in denen er sich über Produkte informiert und sie austestet, und dass er den Kauf dann letztendlich jedoch woanders vollzieht.

Dies ist nicht gewünscht und ist zu vermeiden. Tatsächlich bieten in den hybriden Handel integrierte Showrooms ein großes Potenzial für den Verkauf. Beispielsweise können sie von großen Modemarken gezielt aufgebaut werden, um das Erlebnis "Einkaufen“ so spannend wie möglich für den Kunden zu gestalten. In diesem Punkt kann der deutsche Textileinzelhandel bereits jetzt von anderen Ländern lernen. In London hat Burberry einen Laden realisiert, der darauf ausgelegt ist, dem Kunden vom Betreten des Ladens bis zum Verlassen ein Event zu bieten. Dazu gehören echte und virtuelle Konzerte, Verkäufer, die Informationen zu den Produkten auf TabletPCs zeigen, und virtuelle Spiegel, die Produktinformationen präsentieren. (Burberry 2013).

Eine weitere wichtige Komponente, die derzeit im deutschen Textileinzelhandel fehlt, ist die Einbindung von Social Media am Point of Sale. Seiten, auf denen sich die Menschen im Internet verbinden, um Informationen auszutauschen und in Kontakt zu bleiben, gewinnen im Alltag immer mehr an Bedeutung und nicht selten werden Freunde zuerst via Facebook gefragt, ob die Hose gut aussieht, bevor sie gekauft wird. Ein Pionier diesbezüglich ist C\&A in Brasilien. Dort sind die Kleiderbügel, auf denen die Modewaren hängen, mit dem Internet verbunden, und es wird direkt im Laden angezeigt, wie vielen Personen das Kleidungsstück aktuell gefällt. Somit wird ein wichtiges Maß, das für oder gegen eine Kaufeintscheidung sprechen kann, direkt in den Laden transferiert. (Likes in Echtzeit 2012).

Eine der größten Herausforderungen für den Erfolg des hybriden Handels wird jedoch sein, die Menschen davon zu überzeugen, dass auch und gerade der hybride Handel sorgfältig mit ihren Daten umgeht, dass Verknüpfungen von mobilem, online und stationärem Handel also nicht bedeuten, dass Datenschutzbelange außer Acht gelassen werden. Hier steht speziell der deutsche Textileinzelhandel vor der großen Aufgabe, den sicherheitsorientierten deutschen Kunden zu beweisen, dass hybrider Handel einen Mehrwert ohne unnötige Gefahren bieten kann. Dies kann nur gelingen, wenn hybrider Handel als ganzheitliches, sicheres Konzept implementiert wird, das dem Kunden einen geschützten und zugleich vereinfachten Einkauf im Sinne des Smart Shopping ermöglicht. 


\section{LITERATUR}

Broeckelmann, Philipp 2010: Konsumentenentscheidung im Mobile Commerce, 1. Auflage, Gabler Verlag, Wiesbaden.

Burberry 2013: http://de.burberry.com/store/store-locator/regent-street-store/ o. A., Zugriff am 05.09.2013.

Der Kaufprozess o. 1.: http://wirtschaftslexikon.gabler. de/media/257/222629.png o. A., Zugriff am 08.09.2013.

Esprit Stylingstudio 2013: http://www.esprit.de/stylingstudio o. A., Zugriff am 29.08.2013.

Foscht, Thomas; Swoboda, Bernhard 2007: Käuferverhalten Grundlagen - Perspektive - Anwendungen, 3. Auflage, Gabler Verlag, Wiesbaden.

Jades24 2013: Payment 2013 - Praxiserfahrung aus dem High-End-Fashion E-Commerce mit Schwerpunkt Payment, Frankfurt/Main.

Likes in Echtzeit 2012: http://www.futurebiz.de/artikel/ likes-in-echtzeit-ca-zeigt-gefallt-mir-angaben-in-filialen-an/ o. A., Zugriff am 05.09.2013.

Location-Based-Services 2013: http://wirtschaftslexikon.gabler.de/Definition/location-based-services.html o. A., Zugriff am 04.07.2013.

Marsden, Paul 2013: Digital Point of Sale - Reinventing Retail for the connected Customer. Online verfügbar unter: http://www.syzygy.de/media/21462/syzygy_whitepaper_digital_pos.pdf. Zugriff am 08.09.2013.

Mobile Informationssuche wird für den Einkauf wichtiger 2012: http://www.channelobserver.de/artikel/mobile-informationssuche-wird-fuer-den-einkauf-wichtiger/ o. A., Zugriff am 22.07.2013.

Profitable growth in a digital age - From multi-channel to Total Retail 2013: http://www.pwc.co.uk/retail-consumer/total-retail/introduction.jhtml o. A., Zugriff am 08.09.2013.

Smart Shopping o. J.: http://www.verbraucher. org/1110_eBay_verbraucher.pdf o. A., Zugriff am 21.08.2013.

Stuttgarter Internetagentur LIGADIGITAL 2011: http:// www.openpr.de/news/564279/Stuttgarter-Internetagentur-LIGADIGITAL-ueberarbeitet-den-Online-ShopJADES24.html o. A., Zugriff am 02.09.2013.

Wandeln Sie Ihren Onlineshop 2013: http://www.couchcommerce.com/\#!/de-de/ o. A., Zugriff am 31.08.2013.

Zühlke, Lukas 2013: Einkaufsverhalten in Zeiten des hybriden Handels - Untersuchungen zu notwendigen Anpassungen der Wertschöpfungskette am Beispiel des Textileinzelhandels in Deutschland, Bachelorarbeit, Technische Hochschule Wildau, Wildau.

\section{AUTOREN}

Lukas Zühlke,

B.A. Europäisches Management (TH Wildau)

Consulting Analyst

SHC Stolle \& Heinz Consultants GmbH \& Co. KC

Kleine Johannisstraße 20

20457 Hamburg

I.zuehlke@stolleundheinz.com

\section{Dipl.-Kfm. Michael Stolle}

Geschäftsführender Gesellschafte

SHC Stolle \& Heinz Consultants GmbH \& Co. KG

Kleine Johannisstraße 20

20457 Hamburg

m.stolle@stolleundheinz.com

Prof. Dr. Bertil Haack

Fachbereich Wirtschaft, Informatik, Recht TH Wildau

Hochschulring 1

15745 Wildau

bertil.haack@th-wildau.de 